\author{
3 Research Square

\section{Mei-Lin Wang} \\ Taichung Veterans General Hospital \\ Yu-Pin Chang ( $\sim$ b101091052@hotmail.com ) \\ Taichung Veterans General Hospital \\ Chuan-Han Chen \\ Taichung Veterans General Hospital \\ Ming-Cheng Liu \\ Taichung Veterans General Hospital

\section{Chen-Hao Wu} \\ Taichung Veterans General Hospital \\ Mein-Kai Gueng \\ Taichung Veterans General Hospital \\ Yi-Ying Wu \\ Taichung Veterans General Hospital \\ Jyh-Wen Chai \\ Taichung Veterans General Hospital
}

Preprints are preliminary reports that have not undergone peer review.

They should not be considered conclusive, used to inform clinical practice, or referenced by the media as validated information.

\title{
Relationship Between Clustered Ring Nonmass Enhancement of Breast MRI and Prognostic Molecular Biomarkers in Breast Cancer
}

\section{Research Article}

Keywords: Magnetic Resonance Imaging, Breast Neoplasms, Prognosis, Retrospective studies

Posted Date: January 12th, 2022

DOI: https://doi.org/10.21203/rs.3.rs-1173446/v1

License: (1) (1) This work is licensed under a Creative Commons Attribution 4.0 International License. Read Full License 


\section{Abstract}

\section{Background}

Clustered ring enhancement (CRE) of breast MRI is a lexicon of nonmass enhancement (NME) representing tendency of breast cancer and molecular biomarkers are predictors of response to therapy. The purpose of this study was to retrospectively determine the relationship between CRE NME and prognostic molecular biomarkers in breast cancer.

\section{Methods}

Retrospective analysis of 58 breast lesions in 56 female patients between July 2013 and December 2018 was performed in our institution. Cases with MRI reporting NME in the text were collected via searching the report database. The patterns of enhancement including CRE on breast MRI were reviewed by a radiologist blinded to pathology report. The pathological results and expression of molecular biomarkers were collected. Univariate analysis was applied to evaluate the association between MRI NME imaging features, pathological and IHC stain findings.

\section{Results}

58 Breast lesions were pathologically proven breast carcinoma, and 31 lesions with CRE and 27 lesions without CRE on breast MRI. The expression of estrogen receptor $(E R)(P=0.017)$ and progesterone receptor $(P R)(P=0.017)$ was significantly lower in lesions with $C R E$ compared with those without $C R E$. The expression of Ki-67 ( $\geq 25 \%$ ) was significantly higher in lesions with $\operatorname{CRE}(P=0.046)$. The lesions with CRE have a lower expression ratio of ER $(50.71 \pm 45.39 \%$ vs. $74.26 \pm 33.59 \%, p=0.028)$.

\section{Conclusion}

Our results indicated that lesions with CRE may possess different features from those without CRE in molecular expression. They tend to bear a more aggressive biological behavior.

\section{Introduction}

Clustered ring enhancement (CRE) is a newly-added lexicon of nonmass enhancement (NME) in the 5th BIRADS.

Breast cancer is the most frequently diagnosed invasive cancer in the world and is also the leading cause of cancer death in woman in the developed and high-income countries [1]. Breast cancer is also the most common female cancer with an age-standardized incidence rates of 72.99 per 100,000 persons in 2016 in Taiwan. Mortality rate of breast cancer ranks the third highest among all cancer types in Taiwan. Peak incidence is at 50-59 years old. Although age-standardized incidence rates per 100,000 persons progressively gets higher (as comparing with 49.99 in 2006), the age-standardized mortality rate per 100,000 persons did not significantly increase (10.41 in 2006 vs. 11.68 in 2016) [2, 3]. Good prognosis 
may be resulted from improvement of screening policy, diagnostic tool and treatment planning. Female mammographic screening as public health policy started since 2002 , and some women decided to perform self-paid breast MRI.

Medical advances have transformed the previously surgical-only disease into multidisciplinary approach for breast cancer. Precise diagnosis depends on pathological results of invasive procedure or operative surgery. In some circumstances, reoperation is needed but the patients may hesitate. If noninvasive examination can provide more information in treatment-planning at pre-operative stage, individualized surgical and adjuvant therapy may be set up.

Breast MRI is a useful modality for breast cancer detection, lesion range definition, and staging in newly diagnosed patients with high sensitivity and specificity. Gadolinium enhanced dynamic series are also helpful due to neovascularization in the lesions [4]. In the 4th edition BIRADS, NME was first described as a lexicon group in breast MR, as one kind of the abnormal enhancing breast lesions other than a mass.

According to the 5th BIRADS, clustered ring NME is defined as "thin rings of enhancement clustered together around the ducts." Increasing evidence indicates that clustered ring enhancement correlates with malignant tendency in the recent years $[5,6]$.

There are several credible molecular biomarkers for predicting disease outcomes and systemic treatment effects, including: Expression of estrogen receptor (ER), progesterone receptor (PR), human epidermal growth factor receptor 2 (HER2) and Ki-67.

The treatment planning and the disease prognosis differ greatly depending on the different types of immunohistochemical (IHC) staining results [7].

The purpose of this study was to retrospectively investigate the relationship between CRE NME and prognostic molecular biomarkers in breast lesions.

\section{Material And Methods}

Study population

A retrospective analysis of the MRI database at our institution, a tertiary academic hospital, from July 2013 to December 2018 was conducted. The institutional review committee of Taichung Veterans General Hospital approved the retrospective study and exempted the requirement of informed consent. A total of 56 female patients were enrolled. Two of the patients had bilateral lesions at initial interpretation, and thus 58 lesions of breast carcinoma were recorded and evaluated. The flowchart of patient enrollment is shown in Figure 1.

The criteria for selecting the patients and the lesions were, 
1. (a) We searched our breast MRI database for cases with the keywords of "nonmass like enhancement" or "nonmass enhancement" at the time of initial interpretation.

2. (b) Patient who was pathologically diagnosed with breast cancer or carcinoma in situ and had at least one Immunohistochemistry staining of four selective markers, including Estrogen receptor (ER), progesterone receptor (PR), human epidermal growth factor receptor 2 (HER2), Ki-67

3. (c) Patient who received treatment, such as operation, chemotherapy, radiotherapy, excisional biopsy was excluded.

4. (d) Patient with pathological sampling collected after chemotherapy or hormone treatment was excluded.

MRI protocol

Breast MRI was performed with the patient in a prone position using a 1.5-T commercially available system (Magnetom Aera-Siemens) with a dedicated surface breast coil (16-channel and 18 channel).

Our imaging protocol included a localizing sequence followed by axial fat-suppressed(SPAIR) T2weighted fast spin-echo, and axial spin-echo T1-weighted non-fat-suppressed sequence, DWI/ADC before contrast material administration.

Dynamic T1 weighted fat suppressed 3D gradient-echo sequences (flip angle 12.0 degree; field of view, $320 \times 320 \mathrm{~mm}^{2}$ ) are then performed before and four times after (at approximately 99-second intervals) a bolus intravenous injection of gadobutrol (Gadovist $\circledast$; Bayer Healthcare) $(1.0 \mathrm{mmol} / \mathrm{ml}$ injection) $(2 \mathrm{ml} / \mathrm{s})$ at a dose of $0.1 \mathrm{mmol} / \mathrm{kg}$ body weight in the axial plane.

Other parameters were field-of-view $320 \mathrm{~mm}$, section thickness $1.5 \mathrm{~mm}$, and interslice gaps $20 \%$.

The same imaging protocol is used for both screening and diagnostic indications.

Image interpretation

All images of the total $56 \mathrm{MRI}$ examinations enrolled were reviewed by without information of pathological reports. The patterns of nonmass enhancement on breast MRI were reviewed according to the 5th BIRADS lexicon. The post contrast imaging on the axial, sagittal and coronal plane were evaluated. NME distribution (focal, linear, segmental, regional, multiple regional, or diffuse) and internal enhancement pattern (homogeneous, heterogeneous, clumped, or CRE) were recorded.

The description statistics was conducted for NME pattern and their corresponding pathology.

If the enhancing area had had more than one features, we tended to choose the lexicon of the largest part.

Pathological results 
There were cases initially diagnosed by core needle biopsy and cases by both core needle biopsy and surgery. All tissue samples had been formalin-fixed and paraffin-embedded sections. Histological types were defined according to the World Health Organization classification [8].

The analysis of the expression of molecular biomarkers was performed by IHC staining. IHC staining were performed separately and using monoclonal primary antibodies (Ventana Medical Systems) to estrogen receptor (ER) (SP1), progesterone receptor (PR) (1E2), human epidermal growth factor receptor 2 (HER2) (4B5), and Ki-67.

When the result of HER2 is doubtful, gene amplification is verified by in situ hybridization techniques. Detection procedures followed the manufacturer's instructions for Fluorescence in Situ Hybridization (FISH) kit for the detection of HER2 amplification (Ventana INFORM HER2 Dual ISH DNA probe cocktail assay).

We searched for and recorded the pathological diagnosis and their IHC staining results of ER, PR and Ki67 percentage and FISH of HER2 through reports for every patient with NME on MRI. ER, PR and Ki-67 were recorded as percentage positive tumor nuclei in the sample on testing in the presence of expected reactivity of internal (normal epithelial elements) and external controls. The IHC staining result of HER2 is according to the American Society of Clinical Oncology (ASCO)/College of American Pathologists (CAP) guideline. HER2 positivity was considered as score $3+$ by IHC or FISH positive, whereas cases with score 0 to $1+$ or $2+$ without FISH positive were regarded as negative.

\section{Statistical analysis}

Statistical analyses were carried out using SPSS software, version 19.0, (SPSS, Inc. Chicago, IL, USA).

We performed univariate analysis to evaluate the association between MRI NME imaging features and pathological and IHC stain findings. The association between variables was analyzed using the Pearson chi-square or Fisher exact tests for categorical data and the Student's t-test for continuous data. Variables are found to be significant on univariate analysis $(P$ value $<0.05)$.

\section{Results}

\section{Demographics of the study population and MRI patterns}

We analyzed 58 malignant breast lesions as the study population and its demographics were summarized as Table 1. The ratio of CRE and non-CRE of our study population were 31 cases (53.4\%) and 27 cases (46.6\%), with the latter composed of 17 cases (29.3\%) of clump enhancement, 8 cases (13.8\%) of heterogeneous enhancement and 2 cases (3.4\%) of homogeneous enhancement. CRE was the most common enhancing pattern of our study.

The distribution of the breast lesions was categorized as focal 6 (10.3\%), linear 2 (3.4\%), segmental 19 (32.8\%), regional 10 (17.2\%), multiple regions 17 (29.3\%), diffuse 4 (6.9\%). Segmental and multiple 
regions were the most common enhancing distribution of our study. Figure 2-4 demonstrated typical cases with CRE NME with regional or segmental distribution.

\section{Histological types}

A total of 58 breast lesions were pathologically diagnosed breast malignancy, including $19(32.8 \%)$ as invasive ductal carcinoma(IDC) only, $23(39.7 \%)$ as ductal carcinoma in situ (DCIS) only, $10(17.2 \%)$ as both DCIS with IDC, 4 (6.9\%) as invasive lobular carcinoma or lobular carcinoma in situ and $2(3.4 \%)$ as DCIS (with or without IDC) with lobular cancerization. Pure DCIS was the most common histological types of our study.

The ratio of different NME MRI pattern, distribution and pathological diagnosis were listed in the Table 2.

\section{Age and Demographics}

Mean age of our cases were 48.6 years (29-75 years). For the mean age at initial interpretation, the group of CRE (47.39 \pm 10.58 years) and non-CRE (49.96 \pm 10.63 years) showed no significant difference $(P=0.36)$.

\section{IHC and CRE}

Table 3. and Table 4. showed categorical and quantitative comparison between clustered ring enhancement and prognostic molecular biomarkers in breast cancer.

The statistical results comparing the clustered ring enhancement and the categorical data of prognostic molecular biomarkers of breast cancer were summarized in Table 3. On the other hand, the statistical results of comparing the clustered ring enhancement and continuous data of prognostic molecular biomarkers of breast cancer were summarized in Table 4 .

\section{Association between ER, PR and CRE}

All of the 58 breast lesions had ER and PR staining.

ER showed an expression in $74.14 \%(43 / 58)$ breast lesions among the cases.

PR showed an expression in $65.52 \%(38 / 58)$ breast lesions among the cases.

Expression of ER and PR was significantly lower $(P=0.017, P=0.017)$ in lesions with CRE compared with that without CRE. The breast lesions with CRE pattern tended to exhibit a lower expression ratio of ER than those without CRE pattern $(50.71 \pm 45.39 \%$ vs. $74.26 \pm 33.59 \%, p=0.028)$. The ratio of PR expression of breast lesions with CRE pattern and without CRE pattern exhibited no significant difference $(26.61 \pm 34.65 \%$ vs. $45.74 \pm 41.87 \%, p=0.066)$.

\section{Association between Ki-67 and CRE}


We used Ki-67 level $\geq 25 \%$ as the cut-off point of the Ki-67 proliferative index since better prognostic power was noted according to the previous study [9].

IHC staining of Ki-67 were performed on 38 breast lesions and, of which 17 had high Ki-67 proliferative index. The expression of $\mathrm{Ki}-67(>=25 \%)$ in lesions with CRE was significantly higher $(P=0.046)$ than that without CRE.

The Ki-67 proliferation index of breast lesions with CRE pattern and without CRE pattern exhibited no significant difference $(33.90 \pm 22.41 \%$ vs. $24.33 \pm 23.27 \%, p=0.205)$

\section{Association between HER2 and CRE}

HER2 IHC staining were performed on 39 breast lesions. All of the lesions with results of $2+$ or $3+$ received FISH test. A total of 9 lesions had HER2 positive results. There was no significant difference on HER2 overexpression between lesions with CRE and that without CRE.

\section{Discussion}

- Malignant features of NME

Several studies reported clustered ring enhancement and segmental distribution have the strongest relation with malignancy as compared with other NME features of 5th BIRADS [6, 10-12]. Clustered ring enhancement is considered higher risk of malignancy and more aggressive behavior as compared with clump enhancement, heterogeneous enhancement and homogeneous enhancement [5].

Tozaki et al [6] first provided the concept of clustered ring enhancement of ductal carcinoma in situ in MR image. The study showed segmental distribution, clustered ring enhancement have the highest positive predictive value (PPV), for malignancy, $100 \%$ and $96 \%$, respectively. The specificity of clustered ring enhancement for malignant lesions is $63 \%$ [6]. Other studies conducted by Yang, Lunkiewicz and Chikarmane, respectively, all demonstrated CRE pattern and segmental distribution are significant indicators distinguishing malignant breast lesions.

Hypothesis of formation of CRE

Two hypothesis of clustered ring enhancement are contrast media accumulation in periductal stroma or ductal wall [13] or intraductal wash-in and washout appearance and the scan time is at washout phase [10].

Pathological diagnosis in NME

The most common pathological finding of NME is pure DCIS in our study. The result is compatible with previous studies [13]. On the other hands, most DCIS, about $60-81 \%$ of cases, was interpreted as NME on 
the MRI [14]. NME can also be seen in invasive breast cancer, benign lesions and even normal breast tissue [15].

Common pathological diagnosis in CRE lesions

Uematsu et al [16] reported that 77\% CRE lesions were malignant. Of the malignant lesions, $55 \%$ were DCIS and $45 \%$ were invasive cancers. In the case series of Machida [13], 54\% CRE lesions were invasive cancers and $46 \%$ CRE lesions were carcinoma in situ. Another study reviewed by two radiologists found that CRE was significantly associated with invasive cancer ( $p=0.001$ and $p<0.001$, respectively) but absence of necrosis (both $p<0.001$ ). Interestingly, they mentioned that clumped enhancement was associated with DCIS ( $p=0.025$ and 0.001 , respectively) but presence of necrosis ( $p=0.003$ and 0.001 , respectively) [17].

CRE and biomarkers of ER, PR

ER and PR are two IHC staining markers frequently seen in the breast cancer(75-80\%) [7]. Several studies showed that the higher percentage of ER, PR staining, the less aggressive behaviors of the cancer $[4,18]$. They are also prognostic marker of response to treatment $[4,18,19]$. We found that clustered ring enhancement had significantly negative correlation with the two hormone biomarkers.

Breast cancers can be divided into luminal type(including luminal A and B) and non-luminal type(HER2enriches and triple-negative) [7]. The relationship of non-luminal type breast cancer (with negative ER and PR staining) and clustered ring enhancement on MR images should be further investigated.

Furthermore, the percentage of ER staining has significant difference $(p=0.028)$ between groups with and without CRE (50.71 \pm 45.39 v.s. $74.26 \pm 33.59)$ clustered ring enhancement $(50.71 \pm 45.39)$ and nonclustered ring enhancement (74.26 \pm 33.59$)$. Lower mean value of the percentage of PR staining is noted in the group with CRE cases with clustered ring enhancement NME, but without significance (CRE: $26.61 \pm$ 34.65 vs. non-CRE: $45.74 \pm 41.87, p=0.066)$.

CRE and biomarkers of Ki67

$\mathrm{Ki}-67$ is proved to be proliferative marker and strong prognostic indicator for overall survival and disease free survival $[18,20]$.

Previous study by Lee. et al, has shown high Ki-67 expression may be correlated to CRE pattern ( $p=0.048$ and 0.003 , reviewed by two radiologists) but no correlation between HER2 overexpression and enhancing pattern. The results are similar with our finding [17].

Thus, CRE pattern should be considered as a more aggressive and invasive features of breast lesion.

Limitation

First, it is a retrospective, single-institution study, and the sample size is small. 
Second, there is selection bias in our study: Only cases with lesions that were interpreted as NMEs by a single radiologist at the time of initial interpretation were included in the analyses.

For lesions with cluster ring NME without pathology result, if the lesion cannot be observed on sonography or mammography, their pathology result cannot be acquired due to lack of MR-guided biopsy in our institution.

Third, there was no pathology review for this study, and therefore there may be diagnostic heterogeneity among different pathologists.

\section{Conclusion}

Our results indicated that lesions with CRE on breast MRI were different from that without CRE in molecular expression. It expressed a more aggressive biological behavior.

\section{Abbreviations}

NME- Nonmass enhancement

CRE- Clustered ring enhancement

ER- Estrogen receptor

PR- Progesterone receptor

HER2- Human epidermal growth factor receptor 2

IHC staining- Immunohistochemical staining

\section{Declarations}

\section{Declarations}

-Ethics approval and consent to participate:

The study was approved by Institutional Review Board of Taichung Veterans General Hospital. Individual consent was waived due to the retrospective study design. All methods were carried out in accordance with relevant guidelines and regulations.

-Consent for publication: Not applicable

-Availability of data and materials: The authors declare that all data supporting the findings of this study are available within the paper and its source data for the figures in this study are available from the authors upon request. 
-Competing interests: The authors declare that they have no competing interests.

-Funding: Not applicable

-Authors' contributions: Mei-Lin Wang and Yu-Pin Chang wrote the main manuscript text. Chuan-Han Chen, Ming-Cheng Liu and Mein-Kai Gueng provideds cases and read the images. Chen-Hao Wu suggested the methods for analysis. Yi-Ying Wu and Jyh-Wen Chai provided the technical support for MR examination.

-Acknowledgements: Not applicable

\section{References}

1. Harbeck N, Penault-Llorca F, Cortes J, Gnant M, Houssami N, Poortmans P, Ruddy K, Tsang J, Cardoso F: Breast cancer. Nat Rev Dis Primers 2019, 5(1):66.

2. Bureau of Health Promotion DoHtEY: Cancer registry annual report, 2006. In. Taiwan; 2009.

3. Health Promotion Administration MoHaW: Cancer registry annual report, 2016. In. Taiwan; 2018.

4. Mann RM, Kuhl CK, Moy L: Contrastenhanced MRI for breast cancer screening. J Magn Reson Imaging 2019.

5. Asada T, Yamada T, Kanemaki Y, Fujiwara K, Okamoto S, Nakajima Y: Grading system to categorize breast MRI using BI-RADS 5th edition: a statistical study of non-mass enhancement descriptors in terms of probability of malignancy. Jpn J Radio/2018, 36(3):200-208.

6. Tozaki M, Igarashi T, Fukuda K: Breast MRI using the VIBE sequence: clustered ring enhancement in the differential diagnosis of lesions showing non-masslike enhancement. AJR Am J Roentgenol 2006, 187(2):313-321.

7. Tirada N, Aujero M, Khorjekar G, Richards S, Chopra J, Dromi S, loffe O: Breast Cancer Tissue Markers, Genomic Profiling, and Other Prognostic Factors: A Primer for Radiologists. Radiographics 2018, 38(7):1902-1920.

8. Lakhani SR, Ellis. I.O., Schnitt, S.J., Tan, P.H., van de Vijver, M.J. (ed.): WHO Classification of Tumours of the Breast: IARC Press; 2012.

9. Petrelli F, Viale G, Cabiddu M, Barni S: Prognostic value of different cut-off levels of Ki-67 in breast cancer: a systematic review and meta-analysis of 64,196 patients. Breast Cancer Res Treat 2015, 153(3):477-491.

10. Chikarmane SA, Michaels AY, Giess CS: Revisiting Nonmass Enhancement in Breast MRI: Analysis of Outcomes and Follow-Up Using the Updated BI-RADS Atlas. AJR Am J Roentgenol 2017, 209(5):1178-1184.

11. Lunkiewicz M, Forte S, Freiwald B, Singer G, Leo C, Kubik-Huch RA: Interobserver variability and likelihood of malignancy for fifth edition BI-RADS MRI descriptors in non-mass breast lesions. Eur Radiol 2020, 30(1):77-86. 
12. Yang QX, Ji X, Feng LL, Zheng L, Zhou XQ, Wu Q, Chen X: Significant MRI indicators of malignancy for breast non-mass enhancement. J Xray Sci Technol 2017, 25(6):1033-1044.

13. Machida Y, Shimauchi A, Tozaki M, Kuroki Y, Yoshida T, Fukuma E: Descriptors of Malignant Nonmass Enhancement of Breast MRI: Their Correlation to the Presence of Invasion. Acad Radio/2016, 23(6):687-695.

14. Greenwood HI, Heller SL, Kim S, Sigmund EE, Shaylor SD, Moy L: Ductal carcinoma in situ of the breasts: review of MR imaging features. Radiographics 2013, 33(6):1569-1588.

15. Amornsiripanitch N, Lam DL, Rahbar H: Advances in Breast MRI in the Setting of Ductal Carcinoma In Situ. Semin Roentgenol 2018, 53(4):261-269.

16. Uematsu T, Kasami M: High-spatial-resolution 3-T breast MRI of nonmasslike enhancement lesions: an analysis of their features as significant predictors of malignancy. AJR Am J Roentgenol 2012, 198(5):1223-1230.

17. Lee SM, Nam KJ, Choo KS, Kim JY, Jeong DW, Kim HY, Kim JY: Patterns of malignant non-mass enhancement on 3-T breast MRI help predict invasiveness: using the BI-RADS lexicon fifth edition. Acta Radiol 2018, 59(11):1292-1299.

18. Wan ZB, Gao HY, Wei L, Zhang AQ, Zhang JY, Wang Y, Wang DD, Zhang Y: Expression of estrogen receptor, progesterone receptor, human epidermal growth factor receptor 2, and Ki-67 in ductal carcinoma in situ (DCIS) and DCIS with microinvasion. Medicine (Baltimore) 2018, 97(44):e13055.

19. Lamb CA, Vanzulli SI, Lanari C: Hormone receptors in breast cancer: more than estrogen receptors. Medicina (B Aires) 2019, 79(Spec 6/1):540-545.

20. Inwald EC, Klinkhammer-Schalke M, Hofstadter F, Zeman F, Koller M, Gerstenhauer M, Ortmann O: Ki67 is a prognostic parameter in breast cancer patients: results of a large population-based cohort of a cancer registry. Breast Cancer Res Treat 2013, 139(2):539-552.

\section{Tables}




\begin{tabular}{|ll|}
\hline Table 1: Demographics of the study groups & $56^{\star}$ \\
\hline Patient No. & $\begin{array}{c}48.6 \text { years } \\
(29-75 \text { years })\end{array}$ \\
\hline Mean age & 1 \\
\hline Health exam & 55 \\
\hline Clinics & 35 \\
\hline Operation & 23 \\
\hline NME No. & $58^{\star}$ \\
\hline Immunohistochemical staining No. & \\
\hline ER & 58 \\
\hline PR & 58 \\
\hline Ki67 & 38 \\
\hline HER2 & 39 \\
\hline *Two patients have bilateral malignant NMEs. & \\
NME = Nonmass enhancement, ER = estrogen receptor, PR = progesterone receptor, HER2 = human \\
epidermal growth factor receptor 2.
\end{tabular}


Table 2: Ratio of the 58 malignant breast lesions with different NME MRI pattern, distribution and pathological diagnosis

\section{NME Pattern}

Clustered ring enhancement

Clump enhancement

Heterogeneous enhancement

Homogeneous enhancement
No. (\%)

$31(53.4 \%)$

$17(29.3 \%)$

$8(13.8 \%)$

$2(3.4 \%)$
Focal

Linear

Segmental

Regional

Multiple regions

Diffuse

\section{Pathological Diagnosis}

IDC

DCIS

both DCIS with IDC

LCIS with or without ILC

DCIS (with or without IDC) with lobular cancerization
$6(10.3 \%)$

$2(3.4 \%)$

$19(32.8 \%)$

$10(17.2 \%)$

$17(29.3 \%)$

$4(6.9 \%)$

NME = Nonmass enhancement, IDC = invasive ductal carcinoma, DCIS = ductal carcinoma in situ, ILC = invasive lobular carcinoma, LCIS = lobular carcinoma in situ. 
Table 3: Relationship between CRE and prognostic molecular biomarkers in breast cancer.

\section{Parameters}

ER

Positive

Negative

PR

Positive

Negative

Ki-67

$\geq 25 \%$

$<25 \%$

$8(21.1 \%)$

HER2

Positive

Negative

With CRE
19 (32.8\%)

12 (20.7\%)

$16(27.6 \%)$

15 (25.9\%)

$12(31.6 \%)$

$5(13.2 \%)$

13 (34.2\%)

0.046 *

$p$-value

Without CRE

$24(41.4 \%)$

3 (5.2\%)

$0.017 *$

$22(37.9 \%)$

$5(8.6 \%)$

$0.017 *$

Analyzed using the Pearson chi-square or Fisher exact tests.

$\mathrm{CRE}=$ clustered ring enhancement, $\mathrm{ER}=$ estrogen receptor, $\mathrm{PR}=$ progesterone receptor, $\mathrm{HER} 2=$ human epidermal growth factor receptor 2 .

${ }^{*} p<0.05$ was considered significant

Table 4: Quantitative comparison for prognostic molecular biomarkers and CRE

\begin{tabular}{lllll} 
Parameters & NME features & No. $/$ Total & Mean \pm SD $(\%)$ & $p$-value \\
ER & With CRE & $31 / 58$ & $50.71 \pm 45.39$ & \\
\hline PR & Without CRE & $27 / 58$ & $74.26 \pm 33.59$ & $0.028^{*}$ \\
\hline Ki67 & With CRE & $31 / 58$ & $26.61 \pm 34.65$ & \\
\hline & Without CRE & $27 / 58$ & $45.74 \pm 41.87$ & 0.066 \\
\hline
\end{tabular}

Analyzed using Student's t-test.

$\mathrm{CRE}=$ clustered ring enhancement, $\mathrm{NME}=$ Nonmass enhancement, $\mathrm{ER}=$ estrogen receptor, $\mathrm{PR}=$ progesterone receptor

${ }^{*} p<0.05$ was considered significant 


\section{Figures}

From Jan 2008 to Dec 2018

Breast lesions that had the first time MRI study in our hospital with NME pattern and tissue proved pathological report. ( $n=178)$

\section{Excluded:}

- The location of tissue proof was different from the location of NME on MRI. $(n=71)$

- The breast MR study was performed after therapeutic procedure or treatment course. $(n=39)$

- The IHC staining was not performed. $(n=10)$

Native breast lesions $(\mathrm{n}=58)$ in $\mathbf{5 6}$ female patients

\section{Figure 1}

The flowchart of patient enrollment
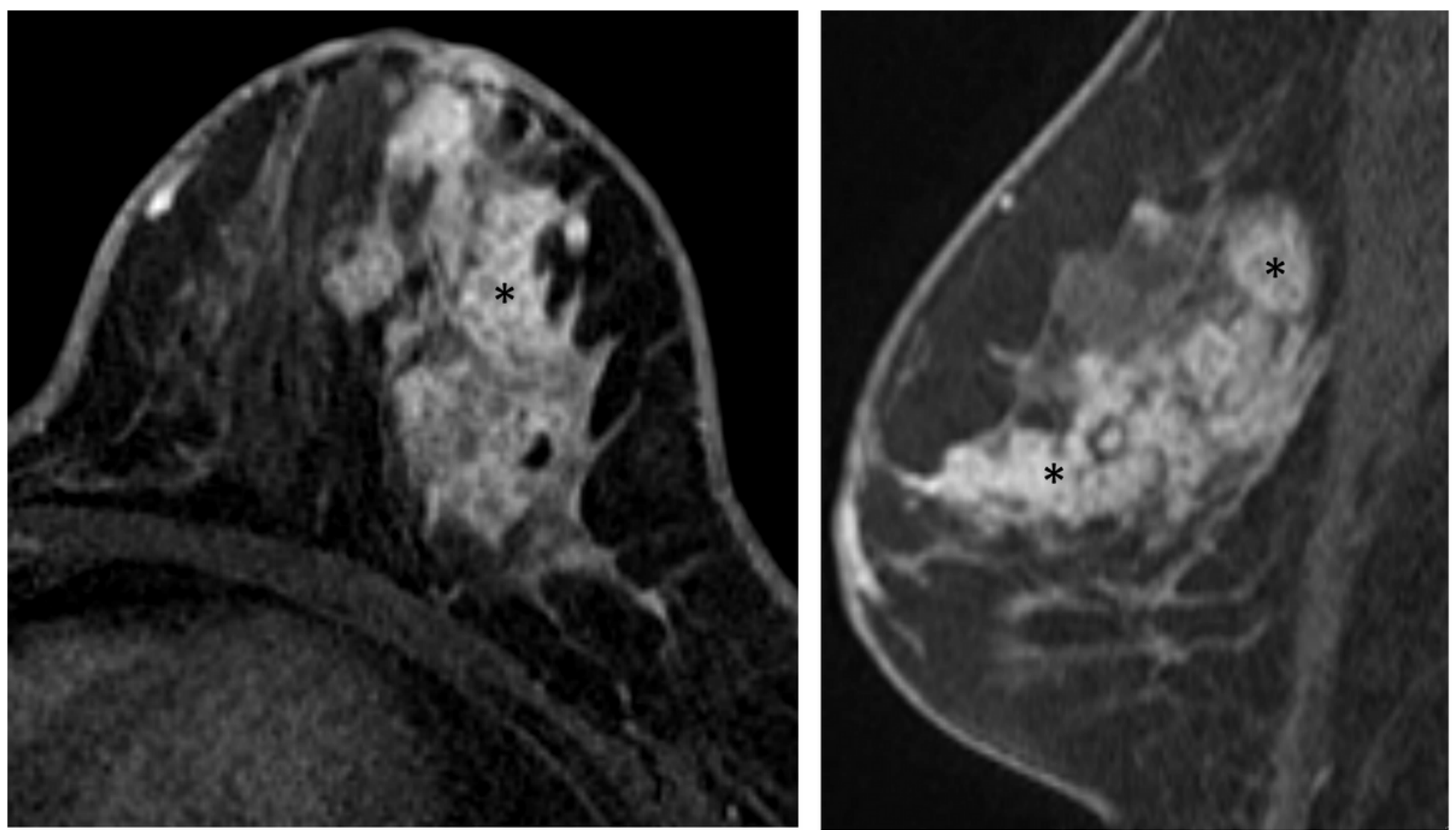


\section{Figure 2}

A 55-year-old woman with left breast palpable lesion for several years and with nipple serous discharge for 2-3 weeks. Axial(a) and sagittal(b) T1-weighted contrast-enhanced subtraction MR imaging shows a clustered ring NME lesion with segmental distribution $(*)$. Pathological results of left breast revealed ductal carcinoma in situ.
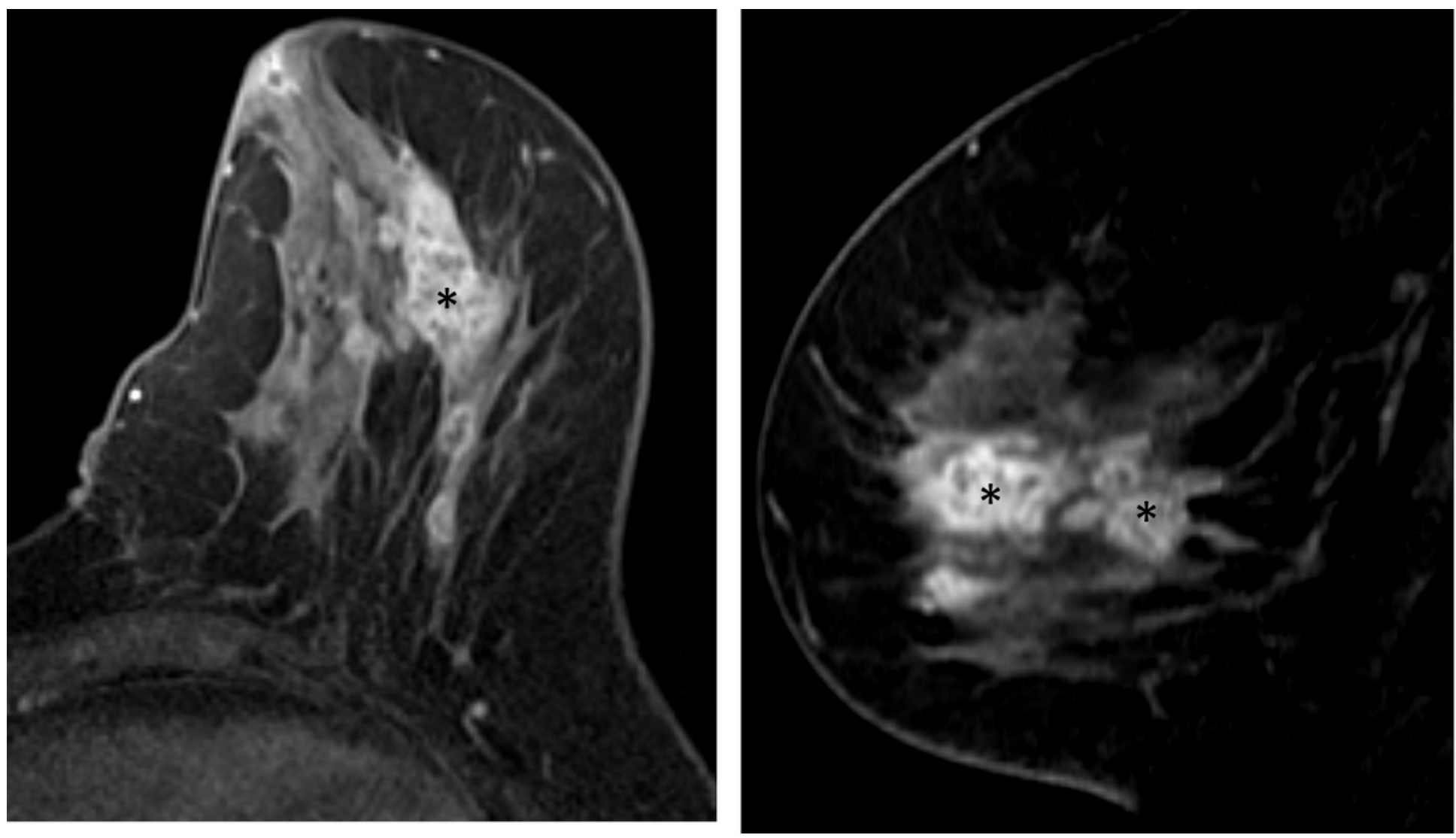

\section{Figure 3}

A 77-year-old woman with left breast mastalgia for one week. Axial(a) and sagittal(b) T1-weighted contrast-enhanced subtraction MR imaging shows a clustered ring NME lesion with segmental distribution (*). Pathological results of left breast revealed infiltrating duct carcinoma and ductal carcinoma in situ. 

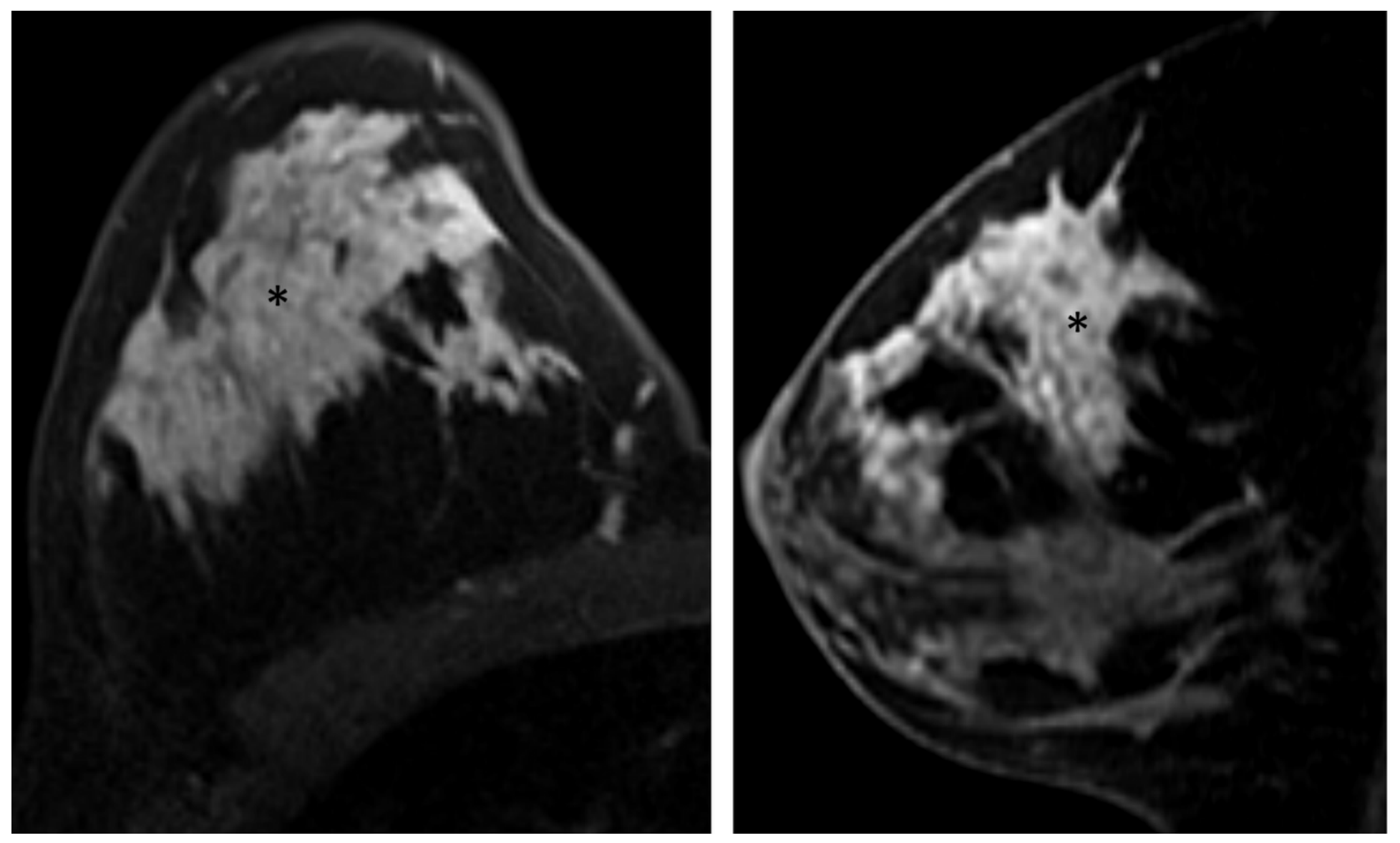

Figure 4

A 36-year-old woman with right breast mass for one month and red nipple discharge. Axial(a) and sagittal(b) T1-weighted contrast-enhanced subtraction MR imaging shows a clustered ring NME lesion in right breast with regional distribution $\left(^{*}\right)$. Pathological results of right breast revealed infiltrating duct carcinoma and ductal carcinoma in situ. 